\title{
Inexpensive microcomputer systems for research and instruction: A dream or reality?
}

\author{
H. J. DURRETT, JR. \\ Southwest Texas State University, San Marcos, Texas 78666
}

\begin{abstract}
This paper presents a detailed introduction to two microcomputer systems useful for research and instruction. The systems are ready for immediate use, fully assembled, and require no knowledge of electronics. They also possess the high-level programming language, BASIC, which can be easily learned by researchers and students. The application of microprocessor technology and mass production has allowed the cost for a single microcomputer to be reduced to about $\$ 600$. At this price, virtually any psychology department or individual researcher can begin to employ computer technology in psychological research and instruction. The hardware specifications, software characteristics, criteria for selection, and possible applications of these systems are considered, with emphasis on use in psychological applications.
\end{abstract}

Computer technology has allowed many dreams to become reality. Newell (Note 1) remarks in a paper entitled "Fairytales," "I see the computer as the enchanted technology. Better it is the technology of enchantment, I mean that quite literally .... There are two essential ingredients in computer technology. First, it is the technology of how to apply knowledge to action, to achieve goals.... The second ingredient is the miniturization of the physical systems that have the ability to intelligent action. Computers are getting smaller, and cheaper, and faster, and more reliable and less energy demanding. Everything is changing in the right direction together. The good things do not trade off against the bad ones. More speed does not mean more dollars. Smaller size does not mean lower reliability. On any given date, these tradeoffs ... do certainly hold. But come back next year and everything is better; smaller, cheaper, faster, more reliable and less energy." In short, computer technology offers "the possibility of incorporating intelligent behavior in all the nooks and crannies of our world, with it we could build an enchanted land" (Newell, Note 1).

Actually, this dream is not far from reality. Since the invention of computers there has been a continual decrease in size and cost and an increase in power. The most significant event has been the development of the microprocessor (McLean, 1974). The speed with which this development has occurred is clear when we note that Sidowski wrote, "their potential influence in psychology is yet to be determined" (1975, p. 193). While recognizing their potential, it was thought "that the psychologist should not become involved with microprocessors" since extensive electronic experience was required (McLean, 1974, p. 157). Now, only 2 years later, at this conference we hear of microprocessors and their significant impact on computing in psychology (Polson, 1978). Microcomputer systems have emerged as an "inexpensive means for applying computer tech- nology to the acquisition and control of behavioral data" (Kehoe, Frei, Tait, \& Gormezano, 1975, p. 183).

Until recently, the cost of the computer was only a part of the overall cost of a computerized psychological laboratory (Sidowski, 1975). The basic units afforded little flexibility without the addition of input/output devices, more memory, hardware interfaces, and software support. Sidowski (1975, p. 193) wrote that "payment of $\$ 20,000$ or more for a beginning system is common." The reality of the typical research laboratory is that there are never enough funds to support all of the research initiatives. The application of computer technology to laboratory research has made the funds which do exist more productive, since a general-purpose apparatus can be constructed in place of many pieces of highly specialized hardware (Bailey \& Polson, 1975).

However, the use of laboratory computers has been an unrealized dream for many researchers. The substantial financial, programming, hardware, and personnel commitments required have limited the extent of their use in psychology. Often, by the time all of the necessary facilities are in place, the researcher is confronted with new problems whose origin is to be found in the solution to the original problem. The complexities of the average system dictate that the researcher become experienced in many skills or hire additional professional staff.

The work of two men, Mr. Chuck Peddle of Commodore Business Machines ${ }^{1}$ and Mr. Steve Leininger of the Tandy Corporation, ${ }^{2}$ has led to the development of two of the world's first mass-produced microcomputers. Mass production resulted in significant cost reductions for a basic system suitable for use by psychologists. These stand-alone microcomputer systems generally include the following components: a keyboard, a cathode ray tube (CRT) display, a central processing unit (CPU), an auxiliary storage unit, a limited amount of memory, a high-level programming language, and an 
external input/output (I/O) interface. Table 1 shows the decrease in computer costs caused by the new PET-2001 made by Commodore and the TRS -80 made by Radio Shack.

It has been possible for the skilled electronics engineer to select and build components with similar capabilities for about $\$ 1,000$, but most psychologists are more interested in doing research than in building computers. For purposes of this discussion, I will focus on only two self-contained commercially available systems, the PET-2001 and the TRS-80.

\section{CHOOSING A MICROCOMPUTER}

In selecting microcomputers such as the PET-2001 or the TRS-80, several variables must be considered. The researcher must decide if these assembled computers are capable of performing all of the appropriate tasks or if a specially constructed device will be required. The researcher must decide if tasks can be performed with a high-level language like BASIC or whether it will require extensive machine-language programming. If a language like BASIC can be used, then many of the needed programs have been written. Hollister (1977) presents an informative primer on microprocessor software that can assist the researcher who must apply the microcomputer to laboratory research using assembly language. If the microcomputer is to be interfaced to other devices, it must be determined whether the computer has an adequate bus structure.

Depending on the tasks to be performed, the researcher may need on-line data and program storage. This is inherent in many systems but there are upper limits to data transfer rate and storage capacity. Thus, for physiological applications, high sampling rates may not be possible. Reliability and serviceability are of importance also. The PET-2001 or TRS-80 have many of

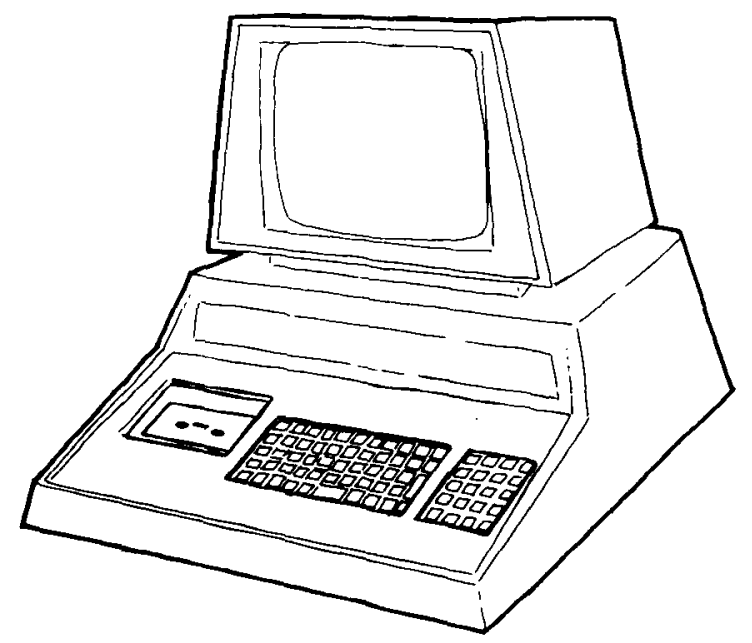

Figure 1. Line drawing of the PET-2001 showing characteristic hardware features.
Table 1

Example of Cost Decreases for a Fixed Hardware Configuration Necessary for Laboratory Research*

\begin{tabular}{lrrrr}
\hline & & & PET- & TRS- \\
& 1972 & 1977 & 2001 & 80 \\
\hline Random Access Memory & 8192 & 8192 & 8192 & 8192 \\
Read-Only Memory & 128 & $* *$ & 14336 & 4096 \\
Keyboard & NA & NA & 73 & 53 \\
Display Size & 1600 & 1024 & 1000 & 1024 \\
Graphics & No & No & Yes & $* * *$ \\
Number of Cassette Drives & 1 & 1 & 1 or 2 & 1 or 2 \\
Resident BASIC Interpreter & No & Yes & Yes & Yes \\
Price (\$) & 11750 & 5400 & 795 & 700 \\
\hline
\end{tabular}

*Adapted from a similar table in NCE/CompuMart PET flyer. **Significant

${ }^{* * *}$ Limited

the characteristics required to perform various tasks in the psychological laboratory.

\section{AN OVERVIEW OF THE PET-2001 AND TRS-80 MICROCOMPUTERS}

\section{The PET-2001: Hardware}

The PET-2001 (Personal Electronic Transactor-2001) is a single-unit microcomputer consisting of a 9-in. diagonal CRT, keyboard, cassette recorder, microprocessor, and associated electronics (Heiser \& Munneche, 1977; Peddle, Note 2). A line drawing of the PET-2001 is shown in Figure 1. The essential hardware specifications of the PET-2001 are presented in Table 2. The microprocessor is a MOS Technology 6502, which is the same microprocessor used in the Apple II color system. Memory for the microcomputer consists of a 14K ROM (read-only memory) and, optionally, either $4 \mathrm{~K}$ or $8 \mathrm{~K}$ RAM (random access memory).

The ROM contains an 8K BASIC language interpreter written by MicroSoft, a $4 \mathrm{~K}$ operating system and a $1 \mathrm{~K}$ machine-language monitor. A $1 \mathrm{~K}$ diagnostic monitor allows the user to identify any hardware malfunction by monitoring several light-emitting diodes (LEDs) contained on the computer's single board. The LEDs indicate a defective component to be replaced.

The video display unit of the PET-2001 is a highresolution 9-in. diagonal black and white CRT. It has a capacity of 1,000 characters arranged in 25 lines of 40 characters or continuous graphics. Although the screen has 64,000 points of resolution, individual points are not plotted; rather, a special character is printed in a character position to activate a particular point. The character set consists of the standard 64 ASCII characters and a selectable 64-symbol graphic set or lowercase alphabetics. All characters can be presented in either a white-on-black or a black-on-white field. Editing is accomplished by a four-directional cursor control which allows any character on the screen to be selectively modified.

The keyboard is like that of Commodore's calculator products. Most users think that it will be inadequate 
Table 2

Hardware Specifications for Commodore PET-2001 Microcomputer

Microprocessor

MOS Technology-6502. All components on a single board. Total unit size and weight (single unit): $42 \mathrm{~cm}$ wide $\times 47 \mathrm{~cm}$ deep $x$ $35.5 \mathrm{~cm}$ high; $20 \mathrm{~kg}$

Memory

$14 \mathrm{~K}$ ROM system consisting of $8 \mathrm{~K}$ ROM BASIC interpreter, $4 \mathrm{~K}$ ROM operating system, 1K ROM machine language monitor, $1 \mathrm{~K}$ ROM diagnostic monitor

Standard: $\quad$ 4K RAM user memory

Optional: $\quad$ 8K RAM user memory

Expansion: To 32K RAM user memory

Video Display Unit

Size: 9-in. diagonal integral high-resolution blackand-white CRT

Capacity: 1,000 characters, 25 lines by 40 columns

Character Set: 64 standard ASCII characters, 64 graphic characters or lowercase characters, normal and reverse field

Cursor and Blinking cursor with four-directional control, Editing: selective character modification, automatic scrolling

Graphics: Graphic characters to draw pictures rather than plot points, continuous graphics with 8 by 8 matrix

Keyboard

Size:

Character Set: 64 ASCII characters available without shift, 64 graphic and reverse field characters available with shift

Form: $\quad 73$ keys total (traditional arrangement), calculator style, 20 keys in numeric pad

Screen Control Clear and erase, character insertion and deleand Editing: tion, line-edit capability, cursor coordinate movement

Special: Run/stop

Input/Output

8-bit parallel user port, IEEE-488 intelligent interface which allows for multiple peripherals, each with device numbers, singlecharacter digital I/O with GET command

On-Line Cassette Storage

1,400-bits/sec Commodore-designed redundant recording scheme, high noise immunity, error detection and correction, uses standard audiotape, second cassette drive interface standard

for typing and, indeed, the skilled two-handed touch typist will find it uncomfortable at first. However, after some experience, a single-hand typing style develops. The keyboard's compact size makes it easy for the hunt-and-peck typist and is especially appropriate for use with children.

The keyboard is not encoded; each key is simply a switch that opens or closes. This characteristic allows the user the option of interfacing a standard-size keyboard if required. Many keyboard kits or assembled packages are available. ${ }^{3}$ With 73 keys, all 64 ASCII characters are available without shifting. A 20-key numeric pad and separate screen control keys provide total keyboard editing capability. A special RUN/STOP key is present on the main keyboard for microcomputer program control.

Input and output for the PET-2001 is accomplished by an 8-bit port and another which conforms to the IEEE- $488^{4}$ interface standard. The 8 -bit interface can be used to interface many existing computer devices to the PET-2001 and the IEEE-488 interface is perhaps the most powerful to appear on a microcomputer to date. There is evidence to suggest this choice was made with the idea that the PET-2001 would be actively used as a laboratory computer. Many electronic devices in the typical chemistry or physics lab have this type of interface. The IEEE-488 interface requires intelligent peripherals but it allows each to be assigned a device number for $\mathrm{I} / \mathrm{O}$ purposes. Further, the multiplexing of signals and interdevice communication is possible. This interface does not allow the PET-2001 to be a terminal; it must always be a controller, although future interface devices will allow communication with other computers. It is possible to accomplish digital $\mathrm{I} / \mathrm{O}$ using the $\mathrm{I} / \mathrm{O}$ interface and the GET command. The GET command allows the microcomputer to wait for specific bit patterns to be received on the $\mathrm{I} / \mathrm{O}$ channel.

All $1 / 0$ for the PET-2001 is accomplished by the monitor. The $8 \mathrm{~K}$ BASIC interpreter contains no $\mathrm{l} / \mathrm{O}$ operations and is currently in initial production as a single chip which will be mass produced and sold to other microcomputer vendors as another product of MOS Technology. ${ }^{5}$

The on-line data storage facility consists of a modified cassette recorder which employs a Commodore-designed high-reliability, redundant recording scheme. Although the unit uses standard audiotapes, it has high noise immunity and error detection and correction capabilities. The cassette recorder is able to transmit and receive data of approximately $1,400 \mathrm{bits} / \mathrm{sec}$.

\section{The TRS-80: Hardware}

The TRS-80 (The Radio Shack Z-80, Note 3) is a four-unit microcomputer consisting of a separate 12 -in. diagonal CRT, keyboard, cassette recorder, and power supply. The Zilog-80 microprocessor and associated electronics are contained in the keyboard unit. A line drawing of the TRS-80 is shown in Figure 2. Table 3 presents a summary of all major hardware specifications of the TRS-80. The TRS -80 memory consists of only $4 \mathrm{~K}$ ROM and optional $4 \mathrm{~K}, 8 \mathrm{~K}$, or $16 \mathrm{~K}$ of RAM within the main board. The $4 \mathrm{~K}$ ROM contains the BASIC interpreter, the keyboard scanning routines, and the video and cassette interface routines. Obviously, the BASIC is limited with only this amount of ROM. It is reported that an $8 \mathrm{~K}$ BASIC will be available by the time this paper is printed. Other features are planned for the hardware but these are beyond the scope of this discussion (Thomas, 1977). 


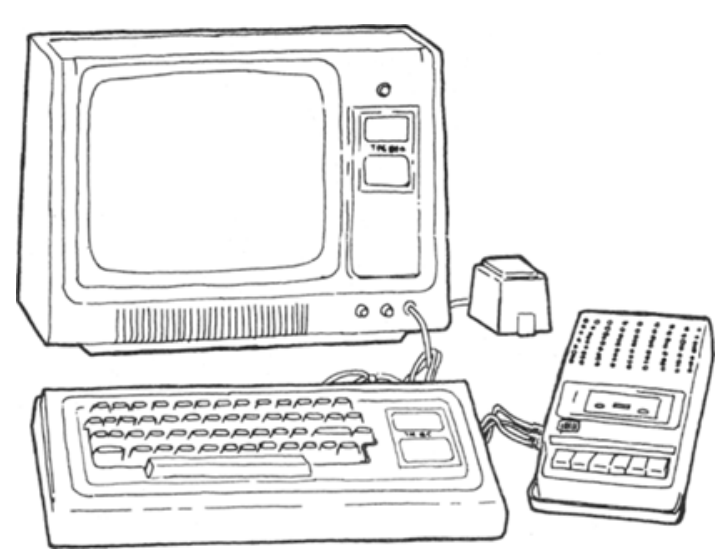

Figure 2. Line drawing of the TRS-80 showing characteristic hardware features.

The TRS- 80 uses a modified black-and-white television set for CRT display. The unit accepts a standard 75-ohm video output with raster scan band width in excess of $6 \mathrm{MHz}$. Any standard video monitor that accepts a 75-ohm input can be used with the TRS-80, but the length of the print line is reduced by one-half. With 1,024 characters arranged in 16 lines of 64 characters, the screen format is like many others currently marketed. The characters are constructed by a 5 by 7 dot matrix in a 6 by 12 cell. Limited graphics are possible by use of three software functions, SET, RESET, and POINT. Each character cell of 6 by 12 dots is divided into 12 blocks of 2 by 3 dots which can be plotted. There are obviously limits to this approach, and the results are only slightly better than line printer graphics.

The keyboard of the TRS-80 is professional and responsive, with a total of 53 keys but only 48 alphanumerics. The interface between the keyboard and microprocessor is accomplished by representing each key on the keyboard as a cross-point or an 8 by 8 matrix. Leininger (Note 4) reports that the "matrix input is driven by the low-order address bits through open collector buffers. The matrix output is sensed by inverting tri-state buffers. Through software manipulation of the address lines, any key closures can be sensed and decoded." The keyboard also has a special reset button located on the back of the keyboard in the auxiliary I/O expansion port. The expansion port is provided for future interface of memory and peripherals. The address bus, data bus, input, output, read, write, interrupt, and interrupt-acknowledge signals are available on a 40-pin connection at the expansion port.

The on-line data storage facility consists of a cassette recorder connected to the TRS- 80 by a read/write line and an on/off line for remote control of the recorder. Recording is accomplished by writing a logical 0 as a single pulse and a logical 1 as a pair of pulses on the unmodified Realistic cassette recorder. The cassette recorder is able to transmit and receive data at approximately $300 \mathrm{bits} / \mathrm{sec}$.

\section{The PET-2001: Software}

Table 4 presents a summary of the PET-2001 software characteristics. The PET-2001 operating system is capable of supporting multiple languages. Theoretically, the language can be incorporated in single chips and used to replace the BASIC interpreter chip in ROM. ${ }^{5}$ The PET-2001 allows the user access to the machine language of the 6502 microprocessor, and it allows machinelanguage access to all monitor $\mathrm{I} / \mathrm{O}$ routines. The file management system is inherent in the operating system.

Table 3

Hardware Specifications for Radio Shack TRS-80 Microcomputer

Microprocessor

Zilog-Z80 (separate unit in keyboard). Total size and weight (four components in unit): keyboard and microprocessor, $42 \mathrm{~cm}$ wide $\mathrm{x}$ $20.3 \mathrm{~cm}$ deep $\times 8.9 \mathrm{~cm}$ high; CRT display, $42 \mathrm{~cm}$ wide $\times 34.3 \mathrm{~cm}$ deep $\times 30.5 \mathrm{~cm}$ high; CTR-41 data cassette, approximately $12 \times 25 \times$ $8 \mathrm{~cm}$; power supply, approximately $6 \times 4 \times$ $4 \mathrm{~cm}$; weight, approximately $27 \mathrm{~kg}$

Memory

4K ROM system contains Radio Shack Level 1 BASIC, keyboard scanning routines, video display drivers, cassette interface routines

Standard: $\quad$ 4K RAM user memory

Optional: $\quad 8 \mathrm{~K}$ or $16 \mathrm{~K}$ RAM user memory

Expansion: Up to $62 \mathrm{~K}$ user memory

Video Display Unit (Separate Unit)

Size: 12-in. diagonal stand-alone black-and-white CRT

Capacity: 1,024 characters, 16 lines by 64 columns Character Set: 5 by 7 dot matrix in a 6 by 12 cell, 48 characters

Cursor and Underscore nonblinking cursor, automatic Editing: scrolling

Graphics: 128 horizontal points by 48 vertical points, 6 by 12 cell divided into 2 by 3 graphable points

Keyboard (Separate Unit)

Size: $\quad$ Typewriter-like keyboard, $41.25 \times 20 \times 8.75 \mathrm{~cm}$ Character Set: 48 characters

Form: 53-key detached keyboard

Screen Control and Editing:

Special:

Clear screen, point set and reset

Reset button in connector slot

Input/Output

Expansion port supplied for more memory and peripherals (address bus, data bus, input, output, read, write, interrupt and acknowledge signals available on a 40-pin card edge connector at rear of keyboard); cassette recorder interface; record and on/off

On-Line Cassette Storage

300-bit/sec ordinary cassette recorder, Logical 0 is a single pulse, Logical 1 is a pair of pulses, file handling by relay to turn recorder on/off 
Table 4

Software Specifications for Commodore PET-2001 Microcomputer

\begin{abstract}
Operating System
Capable of supporting multiple languages, machinelanguage accessibility, file management inherent in operating system, all I/O managed by operating system

BASIC Interpreter

Expanded 8K BASIC written by MicroSoft; $20 \%$ faster than most; strings, integers, and multidimensional arrays; 10 significant digits for high precision; BASIC language control of cursor, reverse field, and graphics; BASIC cassitte file management
\end{abstract}

BASIC Commands

Standard Dartmouth BASIC Statements:

*LET *READ *PRINT *DATA *IF DIM

*THEN *FOR *NEXT DIM *END *GO TO

Extended BASIC Statements:

$\begin{array}{lrlrl}\text { *RESTORE } & \text { REM } & \text { GET } & \text { *GOSUB } & \text { DEF } \\ \text { *RETURN } & \text { *STOP } & \text { *STEP } & \text { *INPUT } & \text { FN } \\ \text { *ON . . . GO TO } & \text { *ON ... GOSUB } & \end{array}$

Scientific Functions: SGN *ABS *RND COS ATN EXP ${ }^{*}$ INT SQR SIN TAN LOG $\pi$

Logical Operations: AND OR NOT

Operation Commands: *RUN *NEW *CLR *LIST *CONT FRE

Formatting Functions:*TAB *POS *SPC

String Functions: LEFT\$ RIGHT\$ MID\$ CHR\$ ASC LEN VAL STR\$

*Indicates statement also in Level 1 BASIC of TRS- 80

Extended 1/O Statements

OPEN and CLOSE logical files on a physical device; SAVE, LOAD, and VERIFY (store and retrieve programs; LOAD allows program overlay; PRINT\#, INPUT \# communication with logical device number; GET\# input one character; CMD multidevice $I / O$ simultaneously

Variables

Types:

REAL; INTEGER (denoted by \%); STRING (denoted by $\$$ ); no limit on number of strings (maximum string length 245 characters)

Names: 962 variable names; letter-letter and letter or digit

\section{Machine-Level Statements in BASIC}

PEEK and POKE-examine and store at a specific memory location; USR and SYS-machine-language subroutines with parameter passing; WAIT-monitor status of a memory location

All I/O data files can be opened or closed at run time by referencing file names of up to 80 characters. Table 5 presents an example of $\mathrm{I} / \mathrm{O}$ operation using these features of the PET-2001.

The BASIC interpreter provided with the PET is approximately $20 \%$ faster than other microcomputer BASICs. It allows for an arbitrary number of strings, integers, or multidimensional arrays. Ten significant digits of accuracy are provided for all calculations. All CRT and keyboard functions are available in BASIC. The summary of BASIC commands found in Table 4 available on the PET-2001 indicates an impressive capability which surpasses most minicomputers and almost all large-computer BASICs. For comparison purposes, those statements also present in TRS -80
BASIC are indicated with an asterisk. In addition to the OPEN and CLOSE extended I/O commands, the user is able to SAVE and LOAD programs as well as VERIFY them from tape. The LOAD command makes it possible to overlay programs of arbitrary size by observing the constraint that the first program loaded must be the largest program loaded.

The PRINT\# and INPUT\# commands allow communication with any of 16 different logical devices over the IEEE-488 interface. Another feature that many programmers have wished for is the capability to send the same I/O message to several devices simultaneously. This can be accomplished by use of the CMD command. The interface between BASIC and machine-language routines is facilitated by use of the USR and SYS, which allow calls to machine-language subroutines and parameter passing from BASIC.

PEEK and POKE allow the user to selectively examine or modify specific memory locations while WAIT can be used to monitor the status of $I / O$ ports. The software capabilities of the PET-2001 represent a sig. nificant advance in the power of microcomputers.

\section{The TRS-80: Software}

The current capabilites of the TRS- 80 software are shown in Table 6 . The TRS- 80 operating system is BASIC. A more sophisticated BASIC is currently under development and should be available when this paper is printed. From the information that I have, there appears to be no capability to access the Z-80 machine language directly from BASIC.

The current BASIC interpreter for the TRS-80 is limited, with only seven-digit internal precision and six-digit displayed precision. Only a single dimensional array and only two string variables limited to 16 characters each are available. This is a rudimentary set of capabilities but a significant accomplishment given the $4 \mathrm{~K}$ size of ROM. Table 6 also shows the entire instruction set of the TRS-80 BASIC as currently implemented. There are no extended I/O statements or machine-level statements in BASIC. Future development of a Level 2

Table 5

An Example of 1/0 Operations on the PET-2001: A Tape-to-Tape File Copy

$\begin{array}{ll}10 & \text { OPEN } 5,1,0, \text { "OLD FILE" } \\ 20 & \text { OPEN 6,2,1, "NEW FILE" } \\ 30 & \text { INPUT \#5, A \$ } \\ 40 & \text { IF ST AND 64 GOTO 70 } \\ 50 & \text { PRINT \#60, A \$ } \\ 60 & \text { GOTO } 30 \\ 70 & \text { CLOSE 5 } \\ 80 & \text { CLOSE 6 }\end{array}$

Note-This program locates "OLD FILE" on Tape 1 and then writes a file header for "NEW FILE" on Tape 2. The program then copies Tape 1 to Tape 2 until it encounters an END OF FILE on the I/O status word "ST," the end of file being indicated by a decimal 64. It then writes an END OF FILE on Tape 2 and closes Tape 1. 
Table 6

Software Specifications for Radio Shack TRS-80 Microcomputer

Operating System

Supports BASIC Level 1 currently; no machine-language accessibility

BASIC Interpreter

Radio Shack Level 1 BASIC; very, very limited strings (2 strings A\$, B \$, 16 characters long, no capability to compare strings $A \$$ and $B \$$ ); only one single-dimensioned array; 6-digit acceptable precision; Radio Shack Level 2 BASIC-second cassette interface, an assembler/editor, disk operating system, expansion unit for PC boards, modems serial and parallel $\mathrm{I} / \mathrm{O}$ devices

BASIC Commands

Standard Dartmouth BASIC Statements:

$\begin{array}{lllll}\text { LET } & \text { READ } & \text { PRINT DATA } & \text { IF } & \\ \text { THEN } & \text { FOK } & \text { NEXT } & \text { END } & \text { GOTO }\end{array}$

Extended BASIC Statements:

RESTORE STEP GOSUB RETURN STOP INPUT ON...GOTO

Scientific Functions: INT MEM . ABS RND

Logical Operations: None
Operation Commands: RUN NEW CLR LIST CONT

Operation Commands: RUN NEW CLR LINT

String Functions: None; only 2 strings, $A \$$ and $B \$$

Graphic Functions: POINT RESET SET

Extended 1/O Statements None

Variables

Types:

REAL, INTEGER, STRINGS $(2, \mathrm{~A} \$$ and

B\$)

Names: $\quad$ A to $Z$ (6 significant digits), $A(n)$ dimensioned array, $A \$$ and $B \$$ strings

Machine-Level Statements in BASIC None

BASIC will increase the software capabilities of the TRS- 80 substantially.

\section{LABORATORY AND INSTRUCTIONAL APPLICATIONS OF THE PET-2001 AND TRS-80}

All of the powerful features of these two inexpensive microcomputer systems would be valueless to psychologists if it were not possible to utilize them effectively in research or instruction. Table 7 presents a comparison of the features of the PET-2001 and the TRS-80 which makes laboratory and instructional usage possible. Depending on the task, the microcomputers may act as dedicated processors or multiprocessors.

The PET-2001 appears to be useful in real-time laboratory research, since it has a real-time clock capable of indicating both clock time and elapsed time within a $1 / 10$-sec accuracy. This is possible through the use of the TI or TI\$ functions. When monitoring external events, the microcomputer can be placed in a wait state using the WAIT command, which allows the user to monitor I/O ports until specified bits arrive from external devices. Responses can be taken from the keyboard using the GET command. Stimuli can be tachistoscopically displayed in $1 / 30 \mathrm{sec}$ or less. More accurate timing can be achieved by using an external clock on the IEEE-488 interface. The PET-2001 can also be used to process responses and stimuli for a number of devices or individuals by use of the CMD instruction and the IEEE 488 interface. Some additional hardware may be required for this application, such as a response buffer to assure that responses are not lost.

In contrast to the PET-2001, the TRS- 80 currently has somewhat limited application in the real-time laboratory. The development of a software clock or the use of an external clock interfaced through the 40-pin I/O bus would make such tasks feasible. Tachistoscopic presentation of stimuli in $1 / 30 \mathrm{sec}$ or less is also possible with the TRS-80. Level 2 BASIC will obviously enhance the TRS-80's research capabilities.

Both microcomputers should find immediate practical and cost-effective use in instructional areas. Computeraugmented instruction is simply a matter of creating the proper displays, program logic, and data structures. Techniques for use of serial file structures for research and instruction like those discussed by Durrett (1974) are applicable to these microcomputer tasks. Further, a simple information management system (e.g., Durrett, 1976) can be implemented to provide significant information structures to manage and monitor instructional processes.

\section{REFERENCE NOTES}

1. Newell, A. "Fairytales." SIGART Newsletter, 1976, 60, 6-7.

2. Peddle, C. The PET-2001 microcomputer. Paper presented at the West Coast Computer Fair. San Francisco, July 1977.

3. TRS-80 Microcomputer system preliminary user's manual. Fort Worth, Tex: Radio Shack, 1977.

Table 7

A Comparison of Real-Time Capabilities of the Commodore PET-2001 and Radio Shack TRS-80 as Applicable to Psychological Research

WAIT command to monitor memory locations or I/O port until specified bits are set TI and TI\$ variable containing the time of day ST-Variable for status of $1 / 0$ operations
Real-time clock accessible in BASIC

\section{PET-2001 Real-Time Capabilities}

ERASE and CLEAR for instantaneous screen blanking

CMD-For communication with multiple $\mathrm{I} / \mathrm{O}$ devices simultaneously PEEK and POKE to examine and store specific memory locations IEEE-488 intelligent peripheral interface

TRS-80 Real-Time Capabilities

Real-time clock will have to be simulated in software Digital 1/O will have to be accomplished over 40-pin interface bus
Clear screen for instantaneous screen blanking

Level 2 BASIC will allow better device monitoring and control through expansion unit 
4. Leininger, S. W. A trip through the Radio Shack TRS-80 microcomputer system. Internal memorandum, Radio Shack, Fort Worth, Texas, 1977 (submitted for publication).

\section{REFERENCES}

Bailey, D. E., \& Polson, P. G. Real-time computing in psychology at the University of Colorado. American Psychologist, 1975, 30, 212-218.

DurRetT, H. J., JR. A multiprogram approach to real-time experimentation. Behavior Research Methods \& Instrumentation, 1974, 6, 259-262.

DURReTt, H. J., JR. A simple information management sys. tem for on-line experimentation. Behavior Research Methods \& Instrumentation, 1976, 8, 157-163.

Heiser, D., \& Munneche, T. Chuck Peddle on the PET computer. Personal Computing, 1977, 5, 30-40.

Hollister, A. L. A primer on microprocessor software. Instrumentation Technology, 1977, 10, 47-52.

Kehoe, E. J., Frei, L. J., Tait, R. W., \& Gormezano, I. On microprocessor based computers. Behavior Research Methods \& Instrumentation, 1975, 7, 183-186.

Mclean, R. S. Microcomputers for experimental psychology. Behavior Research Methods \& Instrumentation, 1974, 6, 155-158.

Polson, P. G. Microprocessors: Their impact on real-time computing in psychology. Behavior Research Methods \& Instrumentation, 1978, 10, 139-147.

SiDowski, J. B. Instrumentation and computer technology: Applications and influences in modern psychology. American Psychologist, 1975, 30, 191-196.

Thomas. W. Radio Shack's $\$ 600$ home computer. Creative Computing, 1977, 5, 94-95.

\section{NOTES}

1. For information on the PET-2001 contact Commodore Business Machines, Inc., 901 California Avenue, Palo Alto, California 94304, U.S.A. [(415) 326-4000] .

2. For information on the TRS-80 contact Radio Shack, 2617 West Seventh Street, Fort Worth, Texas 76107, U.S.A. [(817) 390-3011].

3. For information on keyboards contact Radio Shack or Electronics Warehouse, Inc., 1603 Aviation Boulevard, Department I, Redondo Beach, California 90278, U.S.A. [(213) 376-8005].

4. For information on the IEEE-488 interface contact The Institute of Electrical and Electronic Engineers, Inc., 345 East 47th Street, New York, New York 10017, U.S.A.

5. Personal communication: Ms. Terry L. Laudereau, Software Coordinator, Engineering Department, Commodore Business Machines. 